\section{Is there an effect of perioperative blood pressure on intraoperative complications during phacoemulsification surgery under local anaesthesia?}

PK Agarwal, M Mathew and M Virdi
Department of

Ophthalmology, Hairmyres Hospitals, Lanarkshire Acute Hospitals NHS Trust, East Kilbride, Scotland, UK

Correspondence:

PK Agarwal, Department of Ophthalmology, Tennent Institute of Ophthalmology, Gartnavel General Hospital, 1053 Great Western Road, Glasgow G12 OYN, Lanarkshire, UK Tel: + 4407931325566 ; Fax: + 4401413390431 E-mail: pankaj_eye@ yahoo.com

Received: 25 March 2009 Accepted in revised form: 4 January 2010 Published online: 5 February 2010

Presented as a poster at the Annual Congress of Royal College of Ophthalmologists meeting at Birmingham in May 2005

\begin{abstract}
Purpose The practice of deferring phacoemulsification procedure on recording raised blood pressure (BP) in the immediate perioperative period is based on the perception of increased intraoperative risk. The significance of perioperative BP recordings on the surgical complications during phacoemulsification procedure was evaluated. Setting: Hairmyres Hospitals, Lanarkshire Acute Hospitals NHS Trust. Patients and methods Patients were classified as hypertensive on the basis of the British Hypertension Society Guidelines. BP recordings during preoperative assessment, admission, and 1-hour postoperatively were recorded in 734 hypertensives and 740 normotensives undergoing phacoemulsification procedure. In addition, BP recordings in the holding area before giving local anaesthesia were noted in the 734 hypertensives. Patient's peri- and intraoperative complications during the procedure were noted.
\end{abstract}

Results The mean age was $72 \pm 10.5$ years and $74 \pm 11.6$ years among the hypertensives and normotensives. There was a significant increase in the number of hypertensives who developed isolated systolic hypertension in the holding area $(95 \%$ confidence interval $=2.82, P<0.001)$ where the mean $B P$ was $171.38 / 78.31 \mathrm{~mm} \mathrm{Hg} \mathrm{(} \pm 30.55 / 16.29)$. A total of 21 hypertensives and 18 normotensives developed intraoperative complications during the phacoemulsification procedure. There was no significant difference $(P=0.41)$ in the intraoperative complications between the hypertensives and normotensives. Conclusion Perioperative increase in BP noted in the holding area among hypertensives did not increase the risk of surgical complications during phacoemulsification procedure when compared with normotensives. We recommend that BP should not be routinely measured in the holding area before phacoemulsification surgery under local anaesthesia.

Eye (2010) 24, 1186-1192; doi:10.1038/eye.2010.4; published online 5 February 2010

Keywords: BP; phacoemulsification; perioperative risk; intraocular; complications

Introduction

Systemic hypertension affects one billion individuals worldwide ${ }^{1}$ and is endemic in the western world, particularly in the elderly. ${ }^{2}$ Higher arterial pressure is associated with a higher risk of myocardial infarction, heart failure, stroke, or kidney disease. ${ }^{3}$

In the recent guidelines of the American College of Cardiology and the American Heart Association, systemic hypertension per se is considered only a minor risk factor that does not affect overall perioperative management (for non-cardiac surgery including cataract surgery). ${ }^{4}$ Recent studies also cast considerable doubt that the perioperative outcome is worse in hypertensive than in normotensive patients in most clinical situations. ${ }^{5,6}$ 
Howell et $a l^{5}$ noted a tendency for an increased incidence of perioperative haemodynamic instability, myocardial ischaemia, and cardiac arrhythmias in patients with severe hypertension (systolic blood pressure $(\mathrm{SBP})>180 \mathrm{~mm} \mathrm{Hg}$, diastolic blood pressure (DBP) $>110 \mathrm{~mm} \mathrm{Hg}$ ) undergoing surgery under general anaesthesia. There is thus little evidence between admission arterial pressures of $<180 \mathrm{~mm} \mathrm{Hg}$ systolic or $110 \mathrm{~mm} \mathrm{Hg}$ diastolic and perioperative complications. The position in patients with admission arterial pressures above this level remains unclear.

Cataract is a problem associated with old age, the average age of patients undergoing cataract surgery being $>70$ years. Understandably, these patients have quite a lot of comorbidity including systemic hypertension.

Modern phacoemulsification procedure under local anaesthesia as a day case now allows cataract removal safely and efficiently in a closed chamber within a short space of time. ${ }^{7}$ The role of systemic hypertension is hence less clear in such a perioperative setting when there is no clear evidence that deferring surgery under local anaesthesia in such patient reduces intraoperative risk or that actually lowering the $\mathrm{BP}$ on the deferred date of subsequent surgery thereby improves surgical outcomes. The British Hypertension Society (BHS) advise treatment not on the basis of arterial pressure alone but according to the overall estimate of cardiovascular risk. ${ }^{8}$ Hence the perception that elevated pressure is associated with increased perioperative risk during elective surgery even under a local anaesthetic, and therefore trying to reduce the arterial pressure is not evidence-based approach. ${ }^{9}$ The cancellation of phacoemulsification procedure under local anaesthesia for the sole reason of uncontrolled, raised BP on the day of the operation is hence difficult to justify.

In a recent study by Henderson et al ${ }^{10}$ looking into the incidence and causes of ocular surgery, cancellations in an ambulatory surgical centre reported cardiovascular or uncontrolled hypertension as the single most common cause of last minute cancellation of ocular surgery.

The aim of the study is to prospectively investigate whether the cancellation of cataract surgery on the day of the operation (perioperatively) owing to suspected increased risk of intraocular complications in patients with high BP is justified. We have also tried to assess whether there was a higher risk of developing systemic complications during phacoemulsification procedure under local anaesthesia in patients who have associated high BP.

\section{Materials and methods}

Ethics committee approval was obtained from the Lanarkshire Acute Hospitals audit and guidelines committee. Over a 1-year period between January 2002 and January 2003, a total of 734 hypertensives were enrolled under a single consultant ophthalmologist, in this prospective controlled clinical study. As there are no studies in the past looking into the relationship between systemic hypertension and cataract surgery complication, the sample size calculation for the study is hypothetical but large enough to represent the population and draw conclusions. The previous ocular and past medical history including systemic hypertension, cardiovascular disease, and diabetic status were noted. All patients and controls underwent a thorough preoperative assessment on a date before their cataract operation, which included BP measurement. Patients with known ocular risk factors such as pseudoexfoliation, pre-existing ocular trauma, anterior uveitis, glaucoma, and combined surgery were not included in this series.

Similarly, on the day of surgery, BP recordings were noted on admission and $1 \mathrm{~h}$ postoperatively. Any perioperative or intraoperative complications were recorded for all patients. In addition, BP was recorded for the 734 hypertensives after the patients were taken to the theatre in the holding area before giving local anaesthesia. Similarly, a total of 740 normotensives undergoing phacoemulsification procedures were also enrolled.

For the purpose of the study, the BP reading and classification used as per published guidelines by the BHS. ${ }^{8}$ Classification equates with those of the European Society of Hypertension ${ }^{11}$ and the World Health Organization-International Society of Hypertension ${ }^{12}$ and is based on clinical BP and not values for ambulatory BP measurement (Table 1). If the SBP and DBP fall into two different categories, the higher value should be taken for classification.

Table 1 Classification of blood pressure levels of the British Hypertension Society

\begin{tabular}{lcc}
\hline Category & $\begin{array}{c}\text { Systolic blood pressure } \\
(\mathrm{mm} \mathrm{Hg})\end{array}$ & $\begin{array}{c}\text { Diastolic blood pressure } \\
(\mathrm{mm} \mathrm{Hg})\end{array}$ \\
\hline $\begin{array}{l}\text { Normal } \\
\text { Optimal }\end{array}$ & $<120$ & $<80$ \\
$\quad$ Normal & $<130$ & $<85$ \\
High normal & $130-139$ & $85-89$ \\
Hypertension & & \\
$\quad$ Grade 1 (mild) & $140-159$ & $90-99$ \\
$\begin{array}{l}\text { Grade 2 } \\
\text { (moderate) }\end{array}$ & $160-179$ & $100-109$ \\
$\begin{array}{l}\text { Grade 3 } \\
\text { (severe) }\end{array}$ & $\geqslant 180$ & \\
& & \\
Isolated systolic hypertension & \\
Grade 1 & $140-159$ & $<90$ \\
Grade 2 & $\geqslant 160$ & $<90$ \\
\hline
\end{tabular}


Table 2 Distribution of blood pressure (BP) recordings among the subjects $(n=734)$ and controls $(n=740)$ at pre-assessment, admission, holding area, and at discharge

\begin{tabular}{|c|c|c|c|c|c|}
\hline Time of BP measurement & $\begin{array}{c}\text { Normal } \\
(<139 ;<89 m m \mathrm{Hg})\end{array}$ & $\begin{array}{c}\text { Mild Group } 1 \\
(<160 ;<99 \mathrm{~mm} \mathrm{Hg})\end{array}$ & $\begin{array}{c}\text { Moderate Group } 2 \\
(<180 ;<110 \mathrm{~mm} \mathrm{Hg})\end{array}$ & $\begin{array}{c}\text { Severe Group } 3 \\
(>180 ;>110 \mathrm{~mm} \mathrm{Hg})\end{array}$ & $\begin{array}{c}\text { Isolated systolic } \\
\text { hypertension Grade } 1 \\
(>140 ;<90 \mathrm{~mm} h \mathrm{~h}) \\
\text { and Grade } 2(\geqslant 160 ; \\
\quad<90 \mathrm{~mm} \mathrm{Hg})\end{array}$ \\
\hline Pre-assessment $(n=734)$ & 238 & 128 & 206 & 55 & 107 \\
\hline Controls $(n=740)$ & 219 & 136 & 215 & 42 & 128 \\
\hline Admission $(n=734)$ & 226 & 124 & 209 & 61 & 114 \\
\hline Controls $(n=740)$ & 226 & 137 & 216 & 88 & 73 \\
\hline Holding area $(n=734)$ & 107 & 129 & 213 & 87 & 198 \\
\hline Controls not recorded & - & - & - & - & - \\
\hline Discharge $(n=734)$ & 217 & 131 & 219 & 65 & 102 \\
\hline Controls $(n=740)$ & 198 & 157 & 227 & 52 & 106 \\
\hline
\end{tabular}

At pre-assessment, any patient who was not a known hypertensive but whose BP was noted to be Grade 1 (mild) hypertension 140-159 SBP and or 90-99 mm Hg DBP (DBP), or a known hypertensive patient with BP recordings Grade 2 (moderate) hypertension 160-179 SBP and or 100-109 $\mathrm{mm} \mathrm{Hg}$ DBP or above was referred to their General Practitioner (GP) for BP control. The patient was given a date for cataract operation only when we received confirmation that the BP was well controlled by his GP. Patients were re-listed for cataract surgery only when we received confirmation that the BP was adequately controlled, that is, 130-139 SBP and or 85-89 mm Hg DBP with or without medication. On the day of surgery, patients who were noted to have Grade 3 hypertension at admission and were not known to be hypertensive were cancelled and were re-listed for cataract surgery once BP was controlled.

\section{Perioperative}

On the day of surgery, BP recordings were again noted on admission and classified as per the BHS Guidelines (Table 1). The BP was recorded after the patient was taken to the theatre while in the holding area before giving local anaesthesia, and $1 \mathrm{~h}$ postoperatively. Any perioperative or intraoperative complications were recorded.

\section{Data analysis}

The individual patients were divided into five groups (Table 2) on the basis of the BHS Guidelines.

We compared the BP recordings between the 734 hypertensives and 740 normotensives at pre-assessment, on admission, and $1 \mathrm{~h}$ postoperatively at discharge. Any difference in the BP recordings and the subsequent intraoperative complications was also noted.
Among the 734 hypertensives, any fluctuations between BP recorded while in the holding area before giving local anaesthesia and the BP at pre-assessment, on admission, and $1 \mathrm{~h}$ postoperatively at discharge were also noted.

Further, to identify whether a relationship between BP and intraoperative complication exists, the maximum and minimum SBP and DBP readings were calculated for each hypertensive. The difference between maximum and minimum SBP and DBP for all hypertensives gave a measure of the maximum observed change in both SBP and DBP. The hypertensives were then grouped according to whether or not an intraoperative complication occurred during the phacoemulsification procedure. Any association between perioperative or intraoperative complications was compared with BP recording using a $t$-test. We also assessed whether there was any correlation between intraoperative complications and BP recordings at pre-assessment, admission, in the holding area, and at discharge using a logistic regression analysis. All calculations were performed using SPSS (SPSS Inc, Chicago, IL, USA) 10 software.

\section{Results}

\section{Demographics}

A total of 734 hypertensives and 740 normotensives undergoing phacoemulsification procedure were enrolled in this study. The mean age was $72 \pm 11.5$ years and $74 \pm 10.6$ years among the hypertensives and normotensives. There was no significant difference between the two age groups $(P=0.37)$. There were 298 (41\%) males and $436(59 \%)$ female hypertensives and 312 (42\%) males and 422 (58\%) female normotensives.

In all, 218 (30\%) hypertensives and 207 (28\%) normotensives were without any significant associated 
systemic disease as mentioned below. In all, $138(19 \%)$ and $154(20 \%)$ normotensives were suffering from diabetes. There were a total of $428 / 734(58 \%)$ hypertensives and 412/740 (56\%) normotensives suffering from pre-existing systemic hypertension.

Among the 734 hypertensives, a total of 211/428 had only systemic hypertension, whereas 84 had coexisting diabetes, 34 had renal failure, 67 had associated cardiac disease, and 32 had suffered from stroke in the past. There were 88 hypertensives who suffered from systemic morbidity other than hypertension. Out of the 88 patients, 54 were diabetic, 19 had cardiac disease, and 15 had suffered from stroke.

\section{BP measurement}

Table 2 shows the distribution of BP recordings in hypertensives and normotensives at pre-assessment, admission, holding area, and at discharge.

BP at pre-assessment

The mean BP recorded at pre-assessment was 150.57/ $79.12 \mathrm{~mm} \mathrm{Hg}(\mathrm{SD} \pm 25.21 / 13.71)$ for the hypertensives and $154.32 / 74.59 \mathrm{~mm} \mathrm{Hg}(\mathrm{SD} \pm 22.11 / 19.33)$ for the normotensives. There was no statistically significant difference between the hypertensive and normotensive groups $(P=0.29)$. Out of the 107 hypertensives with isolated systolic hypertension, 28 were noted to have Grade 2 isolated systolic hypertension.

\section{$B P$ at admission}

The mean BP recorded during admission among the hypertensives was $153.60 / 73.31 \mathrm{~mm} \mathrm{Hg}(\mathrm{SD} \pm 28.33$ / 15.45) and among the normotensives it was $155.39 /$ $71.27 \mathrm{~mm} \mathrm{Hg}(\mathrm{SD} \pm 21.49 / 17.92)$. There was no significant difference in the $\mathrm{BP}$ recording between the hypertensive and normotensive groups $(P=0.31)$. Despite referral to GP for tight normotensives at pre-admission and receiving an all clear from the GP regarding BP normotensives, there was no significant difference (normal: $P=0.34$, Group $1 P=0.21$, Group $2 P=0.43$, Group $3 P=0.37$, and isolated systolic hypertension $P=0.34)$ in the number of hypertensives and normotensives (normal: $P=0.49$, Group $1 P=0.51$, Group $2 P=0.62$, Group $3 P=0.27$, and isolated systolic hypertension $(P=0.12)$ in all five groups at admission before phacoemulsification procedure in comparison with pre-assessment stage.

\section{$B P$ in holding area}

The mean BP recorded among the hypertensives in the holding area was $171.38 / 73.31 \mathrm{~mm} \mathrm{Hg}(\mathrm{SD} \pm 28.33 / 15.45)$ A significant number of patients $(n=198)$ developed isolated systolic hypertension in the holding area when compared with admission $(n=107) \mathrm{BP}$ recordings $(95 \%$ confidence interval $=2.82, P<0.001)$. There was no significant difference noted in the other groups.

$B P$ at discharge

The mean BP recorded at discharge for hypertensives who underwent phacoemulsification surgery was $158.49 / 76.75 \mathrm{~mm} \mathrm{Hg}(\mathrm{SD} \pm 18.84 / 9.72)$ and 154.62/ $72.41 \mathrm{~mm} \mathrm{Hg}(\mathrm{SD} \pm 14.27 / 11.38$ ) for the normotensive groups. This was not statistically significant. There were 217 hypertensives who were noted to have a BP of $<139 \mathrm{~mm} \mathrm{Hg}$ SBP and < $89 \mathrm{~mm} \mathrm{Hg}$ DBP when compared with the hypertensives in the holding area $(n=107)$. There was a significant reduction in BP $(95 \%$ confidence interval $=2.93, P<0.001)$ at discharge $1 \mathrm{~h}$ postoperatively.

\section{Perioperative and intraoperative complications}

A total of 21 hypertensives compared with 18 normotensives developed intraoperative complications during the phacoemulsification procedure.

A total of 17 posterior capsular tears occurred during the phacoemulsification procedure in 734 hypertensives. Altogether, 15 hypertensives required intraocular lens placement in the sulcus, whereas in two patients the lens could be placed in the bag. In addition, there was zonular dehiscence noted in four hypertensives. In all 18 normotensives with posterior capsular tears, the lens was placed in the sulcus. There were no other ocular complications noted on both hypertensives and normotensives. In our series, to the best of our knowledge, there were no systemic complications such as cardiac arrest, myocardial infarction, or stroke in both groups because of the local anaesthesia both intraoperatively and postoperatively. There were also no differences in the complications noted between the hypertensives and normotensives (in whom no BP recordings were made intraoperatively) $(P=0.41)$.

We also calculated the difference between maximum and minimum SBP and DBP calculated for all hypertensives for each patient in all four stages of $\mathrm{BP}$ assessment. This gave us a measure of the maximum observed change in both SBP and DBP. The mean change in BP in hypertensives with intraoperative complications $(n=21)$ was $38.76 / 23.29(\mathrm{SD} \pm 16.39 / 11.74)$ and $41.57 /$ 21.31 (SD $\pm 17.21 / 9.47$ ) in hypertensives with an uneventful $(n=713)$ intraocular surgery. There was no significant difference in the SBP $(F=0.337, t=-0.665$, $P=0.56)$ and DBP $(\mathrm{F}=1.047, t=0.848, P=0.39)$ between the hypertensives in the above two groups. In a linear logistic regression model, hypertensives with an increased BP at preoperative assessment $(P=0.54)$, admission $(P=0.47)$, or holding area $(P=0.41)$ did not have an increased risk of intraoperative complications 
during phacoemulsification procedure. Perioperative increase in BP noted in the holding area was not associated with an increase in the risk of surgical complications during phacoemulsification procedure.

Also, there was no significant difference $(P=0.27)$ in the intraoperative complications between hypertensives who were known hypertensive $(n=428)$ patients with or without coexisting medical conditions and nonhypertensive ( $n=306)$ patients. Overall, patients who either had pre-existing raised BP or those who developed a significant increase in perioperative BP at the time of the procedure did not have a significantly higher risk of intraoperative complications.

\section{Discussion}

Existing data do not suggest that postponing surgery even under general anaesthesia to control arterial pressure will improve perioperative cardiac outcome..$^{8,13}$ Modern phacoemulsification techniques for cataract surgery allow safe implantation of foldable intraocular lens through small self-sealing incisions. The procedure can now be efficiently performed usually within a few minutes. In such a situation when phacoemulsification is performed under local anaesthesia, the risk due to raised $\mathrm{BP}$ on the day of the operation either for intraoperative or systemic complications alone may be small. ${ }^{7}$ In our series, to the best of our knowledge, none of our patients developed any systemic complications postoperatively.

In our study, a significant number of hypertensives developed isolated systolic hypertension before cataract surgery followed by a significant reduction of BP recordings in the immediate postoperative period. In two previous series, a similar tendency for the preoperative BPs to increase and the postoperative BPs to decrease was noted. ${ }^{14,15}$ The authors are of the opinion that this increase in BP recordings, immediately on admission or in the holding area, might be an overestimate of the longterm arterial pressure level (referred to as 'white coat' or 'isolated office hypertension'). This is defined as a persistently elevated clinic arterial pressure of $\geqslant 140$ / $90 \mathrm{~mm} \mathrm{Hg}$ in the presence of an average daytime ambulatory arterial pressure reading of $<135 /$ $85 \mathrm{~mm} \mathrm{Hg} .{ }^{16-19}$ It is clear that in many surgical patients, the admission arterial pressure will not equate to the patient's usual arterial pressure. $^{20}$

Recent studies suggest maintaining arterial pressure perioperatively at $70-100 \%$ of baseline and avoiding tachycardia is a key factor in the optimal management of hypertensive surgical patients. ${ }^{17,21}$ In our series, none of the patients developed perioperative systemic complications requiring anaesthetic intervention. The authors are of the opinion that postponing surgery for the purpose of preoperative arterial pressure control must take into account that arterial pressure should be corrected slowly, and that up to 2 months may be required to reverse some of the hypertension-induced cardiovascular changes. We acknowledge the fact that data are lacking to support such practice. $^{22}$

The Joint National Committee on prevention, detection, evaluation, and treatment of high arterial pressure (2003) recognises that the risk of cardiovascular disease doubles with each increment of 20/10 $\mathrm{mm} \mathrm{Hg}$. However, there are no recommendations or guidelines for the perioperative care of the hypertensive patient both under local and general anaesthesia. ${ }^{1}$ The American Heart Association/American College of Cardiology (ACC/AHA) guidelines comment that hypertension (Stages 1 and 2) is not an independent risk factor for perioperative cardiovascular complications. ${ }^{23}$ They suggest that Stage 3 hypertension (SAP $>180 \mathrm{~mm} \mathrm{Hg}$ and/or DAP $>110 \mathrm{~mm} \mathrm{Hg}$ ) should be controlled before surgery. ${ }^{23}$ Howell et al ${ }^{5}$ suggest that patients with mild and moderate hypertension and no evidence of coronary artery disease or end-organ damage may safely undergo surgery under general anaesthesia without delay. They suggest that it is appropriate to defer anaesthesia and surgery where possible in patients with admission arterial pressures consistent with Stage 3 hypertension, especially if there is evidence of target organ damage. However, it must be borne in mind that this recommendation is made on the basis of evidence of perioperative risk for surgery under general anaesthesia. The risk of perioperative morbidity and mortality is further reduced under local anaesthesia.

In previous case studies, uncontrolled hypertension was noted to present an increased risk of orbital haemorrhage during injection of a local anaesthetic and potential of perioperative suprachoroidal expulsive haemorrhage (SCH) (a catastrophic complication). ${ }^{24}$ Another risk factor for $\mathrm{SCH}$ was noted to be a pulse rate greater than 85 beats per min, and therefore special consideration was suggested for highly anxious patients (perhaps sedation) or those with poorly controlled atrial fibrillation. ${ }^{24}$ These reports were in patients who underwent extracapsular and even intracapsular cataract extraction with retrobulbar anaesthesia. ${ }^{25,26}$ In a recent large series of $109 \mathrm{SCH}$ cases in the United Kingdom, older age, taking at least one cardiovascular medication, peripheral vascular disease, hyperlipidaemia, posterior capsule rupture before $\mathrm{SCH}$, and phacoemulsification conversion were the main risk factors noted. Taking cardiovascular medication seemed to be a better predictor of $\mathrm{SCH}$ than hypertension and elevated preoperative SBP. ${ }^{27}$ A study by Lee et $a l^{28}$ identified ischaemic heart disease, heart failure, and renal failure as risk factors for perioperative cardiac complications. In 
our series, there were $428 / 734$ (58.3\%) hypertensives who suffered from pre-existing systemic hypertension of which 211 (49\%) had only systemic hypertension, whereas 84 patients had coexisting diabetes, renal failure $(n=34)$, associated cardiac disease $(n=67)$, and 32 had also suffered from stroke in the past. Despite a large number of our patients suffering from hypertension and coexisting disease, there was no association with intraocular complications or systemic morbidity. Meticulous preoperative assessment to identify associated cardiovascular risk factors and extent of target organ damage may help identify patients at risk of perioperative morbidity and mortality to be appropriately listed. ${ }^{8}$ In our series, $87 / 734$ hypertensives (12\%) of our patients developed severe hypertension immediately before phacoemulsification procedure (holding area) despite being on maximal medical treatment.

The absence of any significant difference in intraoperative complications between the hypertensive and normotensive groups would suggest that BP in the holding area had no impact on the surgical outcome.

The ultimate goal in the treatment of hypertension and associated coexisting disease during modern cataract surgery must be to reduce the risk of cardiovascular events for the individual patient and to avoid intraoperative complications. Hypertension is only one of a number of risk factors for cardiovascular disease. In our series, a significant number of patients developed isolated systolic hypertension in the holding area when compared with admission BP recordings. The BP then resolved when recorded at discharge. Our study suggests that raised perioperative arterial pressures do not result in adverse postoperative outcomes in patients undergoing the phacoemulsification procedure.

\section{Conclusion}

Blood pressure recording at the time of admission or in the holding area does not reflect the true level of patient's hypertensive status and perioperative BP in the holding area does not seem to alter the risk of intraoperative complications during routine phacoemulsification procedure under local anaesthesia. We recommend that patients undergoing cataract surgery under local anaesthesia with phacoemulsification surgery should not be cancelled on the grounds of raised BP in the immediate preoperative period. On the basis of the results of our study, we would like to recommend some simple guidelines as suggested in the flow chart (Figure 1) for the patient with systemic hypertension undergoing phacoemulsification surgery under a local anaesthetic.

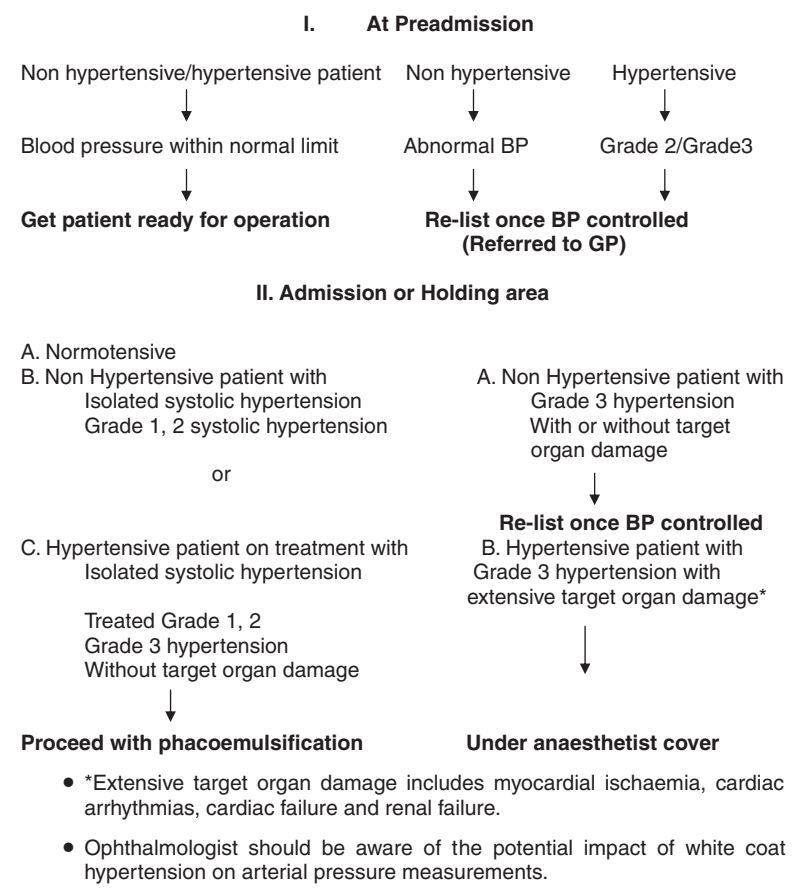

Figure 1 Recommendations for patient with systemic hypertension undergoing phacoemulsification procedure under local anaesthesia.

\section{Summary}

What was known before

- It was not known before whether systemic hypertension associated with increased risk of complication during cataract surgery under local anaesthetic.

What this study adds

- Not every case need to be cancelled due to raised blood pressure for cataract surgery under local anaesthetics.

\section{Conflict of interest}

The authors declare no conflict of interest.

\section{References}

1 Chobanian AV, Bakris GL, Black HR, Cushman WC, Green LA, Izzo Jr JL et al. The seventh report of the Joint National Committee on prevention, detection, evaluation, and treatment of high blood pressure: the JNC 7 report. JAMA 2003; 289: 2560-2572.

2 Priebe HJ. The aged cardiovascular risk patient. Br J Anaesth 2000; 85: 763-778.

3 SHEP Cooperative Research Group. Prevention of stroke by antihypertensive drug treatment in older persons with isolated systolic hypertension. Final results of the Systolic Hypertension in the Elderly Program (SHEP). JAMA 1991; 265: 3255-3264. 
4 Eagle KA, Berger PB, Calkins H, Chaitman BR, Ewy GA, Fleischmann KE et al. ACC/AHA guideline update for perioperative cardiovascular evaluation for noncardiac surgery- Executive summary a report of the American College of Cardiology/American Heart Association Task Force on Practice Guidelines (Committee to Update the 1996 Guidelines on Perioperative Cardiovascular Evaluation for Noncardiac Surgery). Circulation 2002; 105: 1257-1267.

5 Howell SJ, Sear JW, Foëx P. Hypertension, hypertensive heart disease and perioperative cardiac risk. $\mathrm{Br} \mathrm{J}$ Anaesth 2004; 92: 570-583.

6 Chassot PG, Delabays A, Spahn DR. Preoperative evaluation of patients with, or at risk of, coronary artery disease undergoing non-cardiac surgery. $\mathrm{Br} J$ Anaesth 2002; 89: 747-759.

7 Buratto L. Phacoemulsification: Principles and Techniques. Slack: Thorofare, NJ, (1998).

8 Ramsay LE, Williams B, Johnston GD, MacGregor GA, Poston L, Potter JF et al. British Hypertension Society Guidelines for hypertension management 1999: summary. BMJ 1999; 319: 630-635.

9 Stevens A, Abrams K. Consensus, reviews and metaanalysis. In: Stevens A, Abrams K, Brazier J, Fitzpatrick R, Lilford R (eds). Methods in Evidence Based Healthcare. Sage: London, 2001; 367-369.

10 Henderson BA, Naveiras M, Butler N, Hertzmark E, Ferrufino-Ponce $\mathrm{Z}$. Incidence and causes of ocular surgery cancellations in an ambulatory surgical center. J Cataract Refract Surg 2006; 32(1): 95-102.

11 Guidelines Committee. 2003 European Society of Hypertension - European Society of Cardiology guidelines for the management of arterial hypertension. J Hypertens 2003; 21: 1011-1053.

12 Guidelines subcommittee. 1999 World Health OrganizationInternational Society of hypertension guidelines for the management of hypertension. J Hypertens 1999; 17: 151-183.

13 Franklin SS, Jacobs MJ, Wong ND, L'Italien GJ, Lapuerta P. Predominance of isolated systolic hypertension among middleaged and elderly US hypertensives: analysis based on National Health and Nutrition Examination Survey (NHANES) III. Hypertension 2001; 37: 869-874.

14 Suzuki R, Kuroki S, Fujiwara N, Umemoto S. The effects of phacoemulsification cataract surgery via local anesthesia on preoperative and postoperative blood pressure levels. Ophthalmology 1997; 104(2): 216-222.

15 Suzuki R, Kuroki S, Fujiwara N. A comparison of blood pressure changes in phacoemulsification cataract surgery with topical and retrobulbar block local anesthesia. Graefes Arch Clin Exp Ophthalmol 1997; 235(5): 277-282.

16 Pickering TG, Coats A, Mallion JM, Mancia G, Verdecchia P. Blood pressure monitoring. Task force V: white-coat hypertension. Blood Press Monit 1999; 4: 333-341.

17 Kaplan NM. Clinical Hypertension, 8th edn. Lippincott Williams and Wilkins: Philadelphia, 2002, pp 35-36, 92-96.

18 O'Brien E, Coats A, Owens P, Petrie J, Padfield PL, Littler WA et al. Use and interpretation of ambulatory blood pressure monitoring: recommendations of the British Hypertension Society. BMJ 2000; 320: 1128-1134.

19 Pickering TG, Coats A, Mallion JM, Mancia G, Verdecchia P. Blood pressure monitoring. Task force $\mathrm{V}$ : white-coat hypertension. Blood Press Monit 1999; 4: 333-341.

20 Mancia G, Parati G, Pomidossi G, Grassi G, Casadei R, Zanchetti A. Alerting reaction and rise in blood pressure during measurement by physician and nurse. Hypertension 1987; 9: 209-215.

21 Goldman L, Caldera DL. Risks of general anesthesia and elective operation in the hypertensive patient. Anesthesiology 1979; 50: 285-292.

22 Fleisher LA. Preoperative evaluation of the patient with hypertension. JAMA 2002; 287: 2043-2046.

23 Eagle KA, Berger PB, Calkins H, Ewy GA, Fleischmann KE, Fleisher LA et al. ACC/AHA guideline update for perioperative cardiovascular evaluation for noncardiac surgery. A report of the American College of Cardiology/ American Heart Association Task Force on Practice Guidelines (Committee to Update the 1996 Guidelines on Perioperative Cardiovascular Evaluation for Noncardiac Surgery). 2002.

24 Speaker MJ, Guerriero PN, Met JA, Coad CT, Berger A, Marmor M. A case-control study of risk factors for intraoperative suprachoroidal haemorrhage. Ophthalmology 1991; 98: 202-210.

25 Hoffman P, Pollack A, Oliver M. Limited choroidal hemorrhage associated with intracapsular cataract extraction. Arch Ophthalmol 1984; 102: 1761-1765.

26 Manschot WA. The pathology of expulsive hemorrhage. Am J Ophthalmol 1955; 40: 15-24.

27 Ling R, Kamalarajah S, Cole M, James C, Shaw S. Suprachoroidal haemorrhage complicating cataract surgery in the UK: a case control study of risk factors. $\mathrm{Br} \mathrm{J}$ Ophthalmol 2004; 88: 474-477.

28 Lee TH, Marcantonio ER, Mangione CM, Thomas EJ, Polanczyk CA, Cook EF et al. Derivation and prospective validation of a simple index for prediction of cardiac risk of major noncardiac surgery. Circulation 1999; 100: 1043-1049. 